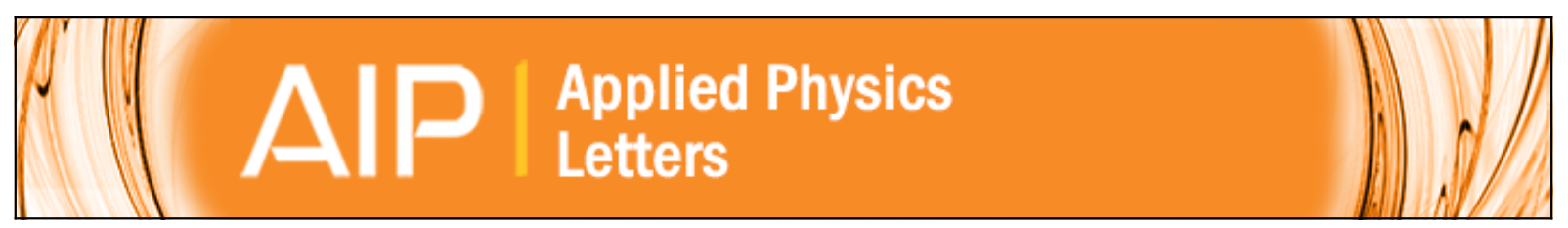

Carrier de-smearing of photoluminescence images on silicon wafers using the continuity equation

S. P. Phang, H. C. Sio, and D. Macdonald

Citation: Applied Physics Letters 103, 192112 (2013); doi: 10.1063/1.4829658

View online: http://dx.doi.org/10.1063/1.4829658

View Table of Contents: http://scitation.aip.org/content/aip/journal/apl/103/19?ver=pdfcov

Published by the AIP Publishing

AlP Re-register for Table of Content Alerts

Create a profile.

Sign up today! 


\title{
Carrier de-smearing of photoluminescence images on silicon wafers using the continuity equation
}

\author{
S. P. Phang, H. C. Sio, and D. Macdonald \\ Research School of Engineering, The Australian National University (ANU), Canberra, ACT 0200, Australia
}

(Received 27 September 2013; accepted 26 October 2013; published online 7 November 2013)

\begin{abstract}
Photoluminescence images of silicon wafers with non-uniform lifetime distribution are often smeared by lateral carrier diffusion. We propose a simple method to de-smear the photoluminescence images by applying the two-dimensional continuity equation. We demonstrate the method on simulated silicon wafers and measured photoluminescence-based lifetime image of multicrystalline silicon wafer. The de-smearing is very effective in recovering the actual lifetime for wafers with gradual changes in lifetime but is less effective around localised recombination centres with high contrast such as grain boundaries and dislocations. The method is sensitive to measurement noise; therefore, the implementation of suitable noise filtering is often critical. (C) 2013 AIP Publishing LLC. [http://dx.doi.org/10.1063/1.4829658]
\end{abstract}

Photoluminescence (PL) imaging ${ }^{1}$ is a powerful method for characterizing silicon wafers. The short acquisition time, high spatial resolution, and contactless nature of the method make it suitable for characterizing wafers in research and also in many stages of production. In principle, a PL image actually represents the distributions of electron and hole concentrations $^{2}$ although it is often converted into an effective minority carrier lifetime image. In doing this, it is usually assumed that lateral diffusion of the minority carriers is negligible. However, when the minority carrier diffusion length is comparable to or larger than the pixel size of the imaging system, the carrier "smearing" effect can become significant. ${ }^{3}$ In samples with non-uniform minority carrier lifetime, this lateral diffusion can result in blurring of the apparent effective lifetime, leading to lower apparent lifetimes in high lifetime regions and vice versa. As the bulk lifetime of multicrystalline silicon is increased through improvement in the casting techniques, ${ }^{4,5}$ the blurring effect of the lateral carrier diffusion is likely to become more significant.

Although the minority carrier distribution after lateral diffusion can be simulated reliably from numerical simulations if the lifetime distribution is known, ${ }^{3}$ it would be more difficult to work backwards from the measured carrier distribution since it would require an iterative fitting of the lifetime at each pixel. In this paper, we propose a simpler, non-iterative method to correct for the lateral carrier diffusion, by applying the 2-dimensional (2D) continuity equation.

The carrier concentration can be calculated directly from the PL intensity with suitable calibration methods. ${ }^{3,6-10}$ We assume here that the minority carrier density is uniform depth-wise and hence the carrier diffusion within the wafer can be simplified into 2D. Despite the non-uniform generation profile, this is a reasonable assumption if the minority carrier diffusion length is larger than the thickness of the wafer and if both surfaces are well passivated. ${ }^{11,12}$ Furthermore, we consider that PL imaging is effectively a steady state measurement since the excitation source is of a constant intensity with exposure time much larger than the effective lifetime. Lastly, we assume that the optical properties and generation rate are uniform across the wafer. In this paper, we will refer to the measured carrier distribution affected by the lateral carrier diffusion and the associated apparent lifetime as "smeared." Similarly, the actual distribution of recombination lifetime that is not affected by lateral carrier diffusion will be referred to as "un-smeared" while the calculated distribution of recombination lifetime that has been corrected for lateral carrier diffusion will be referred to as "de-smeared."

In $2 \mathrm{D}$, the continuity equation can be written as

$$
\frac{d n_{i, j}}{d t}=D\left(n_{i, j}\right) \times \nabla^{2} n_{i, j}+G-\frac{n_{i, j}}{\tau_{i, j}\left(n_{i, j}\right)},
$$

where $i$ and $j$ denotes the position of the point, $n_{i, j}$ is the smeared excess minority carrier concentration, $D\left(n_{i, j}\right)$ is the diffusivity of the minority carrier, $G$ is the generation rate, $\tau_{i, j}\left(n_{i, j}\right)$ is the un-smeared recombination lifetime at the injection level $n_{i, j}$, and $n_{i, j} / \tau_{i, j}\left(n_{i, j}\right)$ represents the recombination rate. The steady state nature of PL imaging infers that $d n_{i, j} / d t=0$, and the equation can be rearranged to determine the carrier recombination lifetime

$$
\tau_{i, j}\left(n_{i, j}\right)=\frac{n_{i, j}}{D\left(n_{i, j}\right) \times \nabla^{2} n_{i, j}+G} .
$$

The $D\left(n_{i, j}\right) \times \nabla^{2} n_{i, j}$ term represents the carrier injections via diffusion from or to neighbouring points which are summed with the calculated $G$ (Ref. 1 ) to estimate an effective local generation rate for every point.

We will first verify the proposed method using simulated wafers because this allows the un-smeared lifetime distribution to be defined and known accurately. Furthermore, the simulated smeared carrier distribution would also be not affected by other smearing effects, such as photon spreading in the silicon CCD and light spread due to light trapping in the imaged sample. ${ }^{13,14}$ As such, the smeared carrier distribution can be known accurately as well. We start by defining the un-smeared lifetime and then numerically simulate the smearing effect of lateral carrier diffusion to obtain the smeared carrier distribution. The simulated smeared carrier distribution is checked by ensuring that $d n / d t \ll G$ and that 
steady state conditions have been reached at every node. Two types of lifetime distribution are considered here.

The first simulated wafer (see Figure 1(a)) is based on a monocrystalline silicon wafer contaminated by a metal particle during high temperature processing,${ }^{15}$ and it represents wafers with gradual changes in lifetime. The contamination source is assumed to be in the centre of the $8.3 \mathrm{~mm} \times 8.3 \mathrm{~mm}$ square wafer. The metal contamination and recombination activity decreases radially in proportion to the complementary error function, with a diffusion length of $1.4 \mathrm{~mm}$. The lifetimes in the uncontaminated region and in the centre of the contamination are $250 \mu$ s and $10 \mu$ s, respectively, and are set to be injection independent. The simulated wafer is of $\mathrm{p}-$ type doping with acceptor concentration of $1 \times 10^{16} \mathrm{~cm}^{-3}$ while the generation rate is set to be $3 \times 10^{18} \mathrm{~cm}^{-3} \mathrm{~s}^{-1}$. The node size used in the simulation is $23 \mu \mathrm{m}$, which is approximately the same as the pixel size of the BT Imaging LIS-R1 system when used with a certain optical magnification lens. The carrier mobility model from Klaassen ${ }^{16,17}$ is used to calculate the effective carrier diffusivity, for both the carrier diffusion model and the subsequent de-smearing calculations. The carrier concentration is assumed to be uniform depth wise, and only the lateral carrier diffusion is considered in the simulation. After simulating the lateral carrier diffusion, random normally distributed measurement noise is added to the smeared carrier distribution, with the standard deviation of the measurement noise estimated from repeated measurements to be $0.5 \%$ of the strongest signal measured.
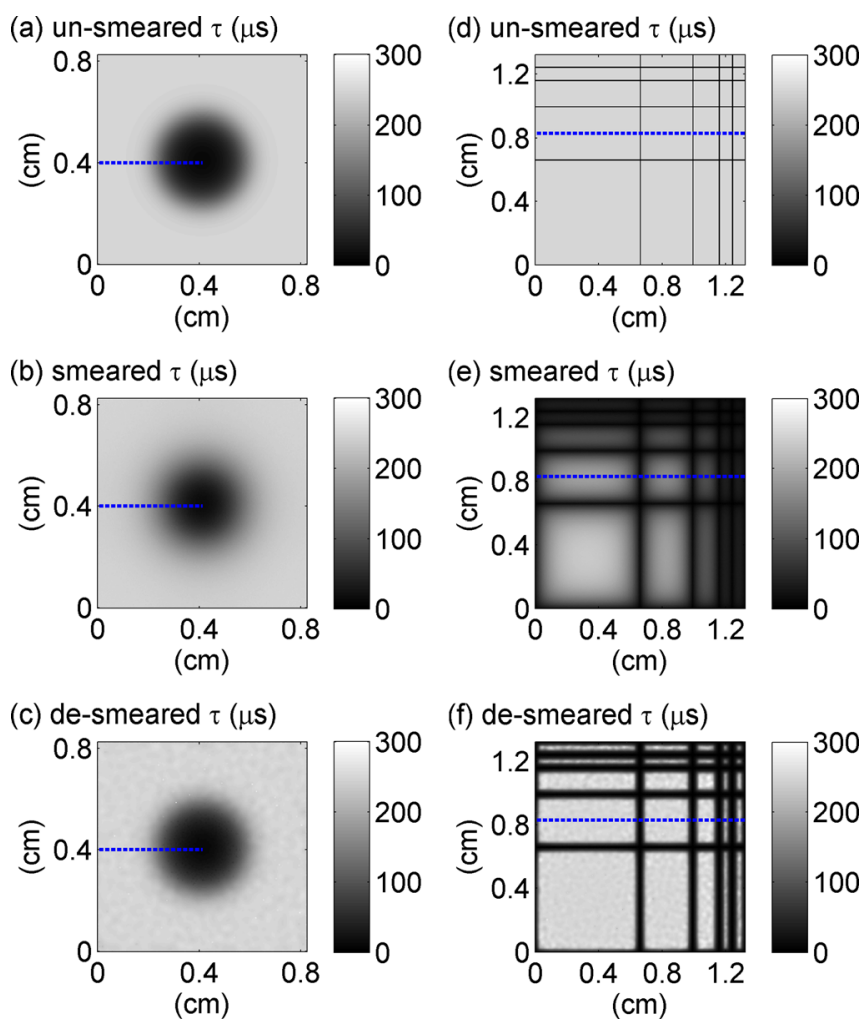

FIG. 1. Demonstration of carrier de-smearing (a)-(c) on the first simulated wafer and (d)-(f) on the second simulated wafer. (a), (d) The un-smeared injection independent lifetime, (b), (e) the smeared apparent lifetime that was simulated numerically, and (c), (f) the de-smeared lifetime that was extracted from the smeared lifetime with the method proposed, using diffusion smoothing. Cross sections of the dotted blue lines are included in Figure 2. Note that the edges of the second simulated wafer in (d) are defined as grain boundaries.
The second simulated wafer is based on a simplified multicrystalline silicon wafer with recombination dominated by grain boundaries, as shown in Figure 2(a). In contrast to the first simulated wafer, the recombination centres are localised, and the changes in lifetime are more sudden. The intra grain regions and grain boundaries are modelled as having injection independent lifetimes of $250 \mu \mathrm{s}$ and $0.25 \mu \mathrm{s}$, respectively. This corresponds to an average intra-grain diffusion length of $0.82 \mathrm{~mm}$. A range of grain sizes are simulated by varying the distance between the grain boundaries from $0.8 \mathrm{~mm}$ to $6.6 \mathrm{~mm}$ while the width of the grain boundaries are kept as 1 pixel. The rest of the properties, including the node size, acceptor concentration, generation rate, and carrier mobility models, are the same as the first simulated wafer.

In addition to simulated wafers, the de-smearing method was also applied on a multicrystalline silicon wafer imaged using the BT imaging LIS-R1 system. The wafer is $140 \mu \mathrm{m}$ thick and of p-type doping with resistivity of $1.6 \Omega \mathrm{cm}$. Both sides of the wafer were passivated using Plasma Enhanced Chemical Vapor Deposition (PECVD) amorphous silicon nitride layers with a surface recombination velocity of less than $10 \mathrm{~cm} \mathrm{~s}^{-1}$. The generation rate during the measurement was calculated to be $7 \times 10^{18} \mathrm{~cm}^{-3}$. An optical magnification lens with a short pass filter (cut-off wavelength of $1050 \mathrm{~nm}$ )

(a)

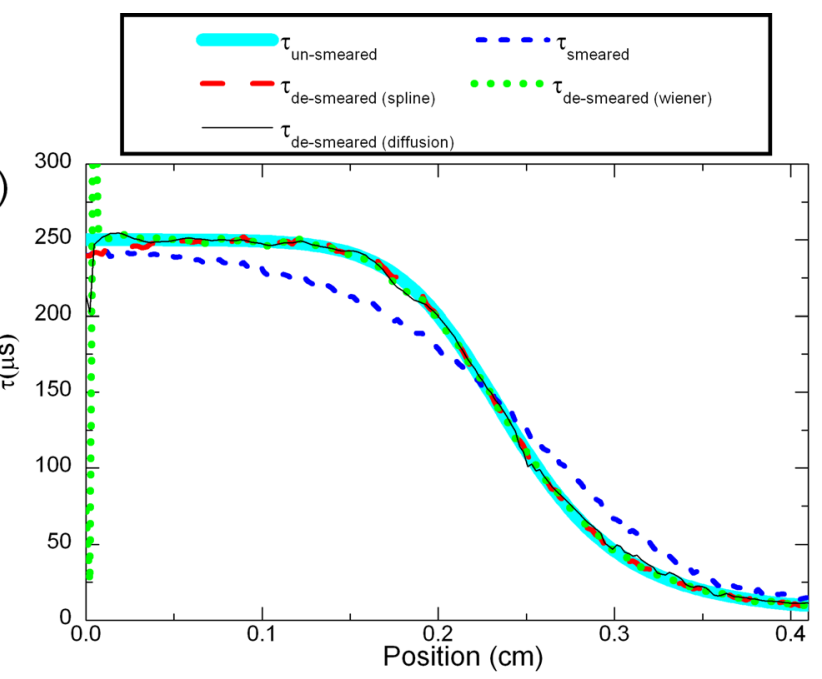

(b)

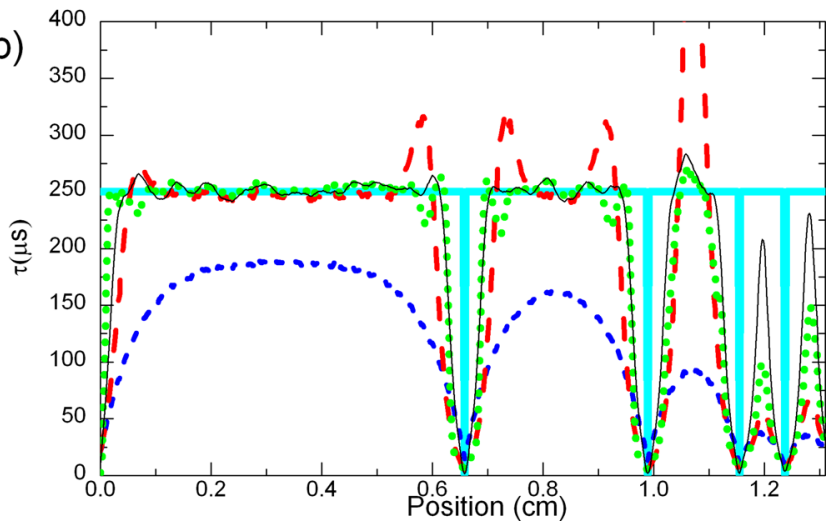

FIG. 2. Cross section of the simulated wafer across the dotted blue lines in Figure 1, showing the un-smeared, smeared, and de-smeared lifetime, (a) for the first simulated wafer and (b) for the second simulated wafer. Note that only half of the wafer is shown in (a) due to the symmetry in the wafer. 
was used during measurement to minimise smearing due to light spread within the imaged sample and photon capture in the Si CCD. ${ }^{9,14,18}$ The smearing due to the Si CCD is further reduced by deconvoluting the image using an experimentally determined point spread function for the system. ${ }^{13,14}$

Since the calculation of the de-smeared lifetime in Eq. (2) is dependent on the estimation of the second derivative, the method is sensitive to measurement noise and the PL images usually have to be filtered before calculating the second derivative. Unfortunately, filtering may also smooth out smaller features, in addition to removing the high frequency measurement noise. Thus, a careful selection of the filtering methods and parameters is required, and in general the choice of these depends on the signal to noise ratio of the measurement system, and also on the nature of the samples being measured. In this paper, we have implemented smoothing splines, ${ }^{19-22}$ a wiener filter, ${ }^{23,24}$ and non-linear diffusion smoothing ${ }^{25}$ in MATLAB for noise filtering.

For the imaged sample, the selection of the noise filtering methods and parameters is more complicated since the actual un-smeared lifetime is unknown, and the amount of smoothing or filtering required may be difficult to determine. In this work, so-called "diffusion smoothing" is used for the imaged sample because of the reduced blurring around grain boundaries, as demonstrated below. Note that diffusion smoothing is simply an alternative method to filter out measurement noise and should not be confused with the real lateral diffusion of minority carriers which we are attempting to correct for. It smoothes the dataset by numerically simulating lateral diffusion for a very short period of time (much shorter than the recombination lifetime). In addition to limiting the diffusion time for the diffusion smoothing, excessive smoothing is controlled by adjusting the diffusion coefficients locally. The diffusion coefficients are decreased when the magnitudes of the residuals between the smoothed and measured data exceed the threshold, which is set based on the measurement noise.

In the case of the first simulated wafer, Figure 1(b) shows that the lateral carrier diffusion has smeared the transition between the edge and centre of the wafer slightly. However, the smearing effect is not very significant, because of the gradual changes in lifetime. Nonetheless, the de-smearing is effective and the de-smeared lifetime is within $10 \%$ of the unsmeared lifetime for most of the region. Fig. 2(a) shows that all 3 of the noise filtering methods considered work well for samples with such gradual changes in lifetime.

Figure 1(e) shows that the apparent smeared lifetime of the second simulated wafer is decreased significantly in the intra grain region, particularly in the smaller grains. The smeared lifetime is decreased to less than $50 \mu \mathrm{s}$ within the $800 \mu \mathrm{m}$ wide grains. It should be noted that the un-smeared lifetime distribution used in this paper is only a simplified model of a multicrystalline wafer, chosen to highlight the effect of lateral carrier diffusion. The lower lifetime within smaller grains in actual multicrystalline silicon wafers could also be caused by injection dependent lifetimes or variations in recombination centre density. After applying the de-smearing calculations, Figure 2(b) shows the lifetime is mostly recovered in the intra grain regions and the de-smeared lifetime is comparable to the un-smeared lifetime. Nonetheless, the de-

smearing is less effective very close to the grain boundaries and in smaller grains, where the signal to noise ratio is weaker. Comparing the noise filtering methods considered, Fig. 2(b) shows that they are comparable far away from the grain boundaries. However, the wiener filter and smoothing splines have a tendency to blur the carrier distribution around the grain boundaries, leading to an apparent widening of the grain boundaries in the de-smeared images. This effect can be reduced for smoothing splines if the smoothing splines are fitted within each grain separately although this is more cumbersome.

Figure 3 shows the de-smearing of the multicrystalline silicon wafer measured using PL imaging, when using diffusion smoothing for noise filtering. The recombination activity of the grain boundaries appear more localised after desmearing and the lifetime within the grains also appear more uniform. Although an injection independent lifetime has been assumed for the simulated wafers, in general, the lifetime of an imaged wafer is injection dependent, and the desmeared lifetime $\left(\tau_{i, j}\left(n_{i, j}\right)\right)$ is only valid for the local injection levels of that measurement, which is given by the smeared PL image. Hence, care should be taken during analysis of the lifetime image because the injection level varies within the PL image. The bright lines on the de-smeared image (see blue dotted circle in Figure 3) can also be observed

(a) smeared $\tau$ ( $\mu \mathrm{s})$

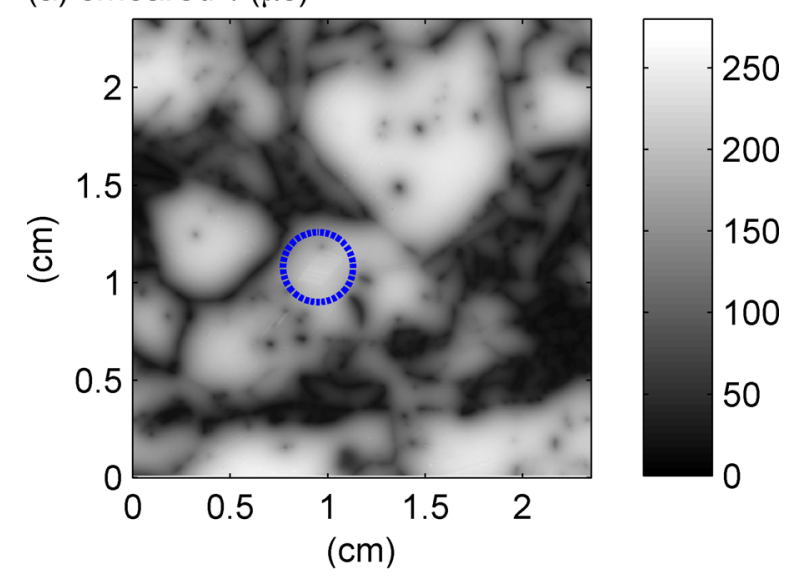

(b) de-smeared $\tau(\mu \mathrm{s})$

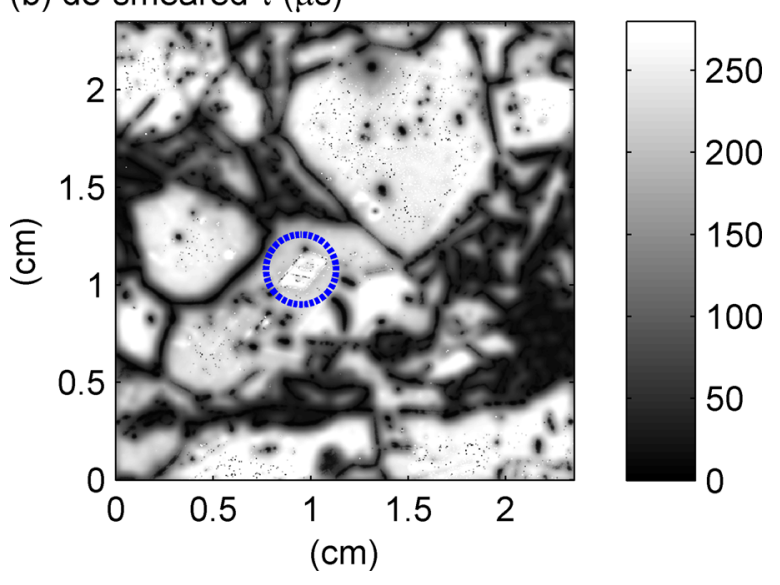

FIG. 3. (a) Lifetime image of a multicrystalline silicon wafer, as measured and (b) de-smeared lifetime with diffusion smoothing. The brighter subgrain boundaries within the blue dotted circles are likely due to optical artefacts. 
under the optical microscope and are likely due to an optical artefact around grain boundaries that resulted in stronger PL signals, ${ }^{26}$ which were amplified by the second derivative during de-smearing. Similar to the simulated wafer, the de-smearing around the grain boundaries and dislocations are expected to be less effective and less accurate. The de-smearing around the grain boundaries can be improved by applying less smoothing. However, the fitting of the carrier concentration should be checked carefully in this case. The fitting can be checked more easily if the de-smearing is to be applied only in 1 dimension, e.g., for a cross section across a grain boundary.

In summary, the effects of lateral carrier diffusion can be significant when the diffusion length of the sample is comparable to the pixel size of the imaging system. We propose a method to correct for the smearing effect using the continuity equation. Application on simulated wafers shows that the lifetime is comparable to the un-smeared lifetime after de-smearing although the method is less effective around high-contrast features such as grain boundaries. It is worth noting that the lateral carrier diffusion is a real effect which occurs during normal solar cell operation, and hence the smeared lifetime may be a more suitable measurement in some cases, providing the impact of additional lateral conduction mechanisms such as the presence of diffused regions is included. However, the de-smeared lifetime allows better accuracy and resolution in studies on recombination centre distributions, such as in interstitial Fe imaging ${ }^{15}$ or boronoxygen defect imaging. ${ }^{27}$ Since the de-smearing method is very sensitive to measurement noise, the selection and implementation of the noise filtering is very critical and will be dependent on the measurement system and the samples being measured. If the other smearing effects from the photon spread in the silicon CCD and the light spread in the wafer are taken into account, the de-smearing process proposed here can significantly improve the accuracy of high contrast PL-based carrier lifetime images.

This work was supported by the Australian Research Council and the Australian Renewable Energy Agency.
${ }^{1}$ T. Trupke, R. A. Bardos, M. C. Schubert, and W. Warta, Appl. Phys. Lett. 89, 044107 (2006).

${ }^{2}$ W. Van Roosbroeck and W. Shockley, Phys. Rev. 94, 1558-1560 (1954).

${ }^{3}$ B. Mitchell, J. Greulich, and T. Trupke, Sol. Energy Mater. Sol. Cells 107, 75-80 (2012).

${ }^{4}$ N. Usami, I. Takahashi, K. Kutsukake, K. Fujiwara, and K. Nakajima, J. Appl. Phys. 109, 083527 (2011).

${ }^{5}$ K. M. Yeh, C. K. Hseih, W. C. Hsu, and C. W. Lan, Prog. Photovoltaics 18, 265-271 (2010).

${ }^{6}$ T. Trupke and R. A. Bardos, in Proceedings of 31st IEEE Photovoltaic Specialists Conference, New York, 2005 (IEEE, 2005), pp. 903-906.

${ }^{7}$ J. A. Giesecke, M. C. Schubert, D. Walter, and W. Warta, Appl. Phys. Lett. 97, 092109 (2010)

${ }^{8}$ J. A. Giesecke, M. Kasemann, and W. Warta, J. Appl. Phys. 106, 014907 (2009).

${ }^{9}$ P. Wurfel, T. Trupke, T. Puzzer, E. Schaffer, W. Warta, and S. W. Glunz, J. Appl. Phys. 101, 123110 (2007).

${ }^{10}$ S. Herlufsen, J. Schmidt, D. Hinken, K. Bothe, and R. Brendel, Phys. Status Solidi (RRL) 2, 245-247 (2008).

${ }^{11}$ R. A. Sinton, A. Cuevas, and M. Stuckings, in Proceedings of 25th IEEE Photovoltaic Specialists Conference, New York, 1996 (IEEE, 1996), pp. 457-460.

${ }^{12}$ D. E. Kane and R. M. Swanson, in Proceedings of 18th IEEE Photovoltaic Specialists Conference, New York, 1985 (IEEE, 1985), pp. 578-583.

${ }^{13}$ B. Mitchell, J. W. Weber, D. Walter, D. Macdonald, and T. Trupke, J. Appl. Phys. 112, 063116 (2012).

${ }^{14}$ D. Walter, A. Y. Liu, E. Franklin, D. Macdonald, B. Mitchell, and T. Trupke, in Proceedings of 38th IEEE Photovoltaic Specialists Conference, New York, 2012 (IEEE, 2012).

${ }^{15}$ S. C. Baker-Finch and K. R. McIntosh, IEEE J. Photovoltaics 1, 66-71 (2011).

${ }^{16}$ D. B. M. Klaassen, Solid-State Electron. 35, 953-959 (1992).

${ }^{17}$ D. B. M. Klaassen, Solid-State Electron. 35, 961-967 (1992).

${ }^{18}$ B. Michl, M. Rudiger, J. A. Giesecke, M. Hermle, W. Warta, and M. C. Schubert, Sol. Energy Mater. Sol. Cells 98, 441-447 (2012).

${ }^{19}$ E. T. Whittaker, Proc. Edinb. Math. Soc. 41, 63-75 (1922).

${ }^{20}$ I. J. Schoenberg, Proc. Natl. Acad. Sci. U.S.A. 52, 947-950 (1964).

${ }^{21}$ C. H. Reinsch, Numer. Math. 10, 177-183 (1967).

${ }^{22}$ C. de Boor, in A Practical Guide to Splines, edited by J. E. Marsden and L. Sirovich (Springer-Verlag, New York, 2001), Vol. 27, pp. 207-242.

${ }^{23} \mathrm{~N}$. Wiener, Extrapolation, Interpolation and Smoothing of Stationary Time Series (MIT Press, Cambridge, 1949).

${ }^{24}$ J. S. Lim, Two-Dimensional Signal and Image Processing (Prentice Hall, Englewood Cliffs, 1990), p. 548.

${ }^{25}$ J. Weickert, Anisotropic Diffusion in Image Processing (B.G. Teubner, Stuttgart, 2008).

${ }^{26}$ A. Y. Liu, D. Walter, S. P. Phang, and D. Macdonald, IEEE J. Photovoltaics 2, 479-484 (2012).

${ }^{27}$ S. Y. Lim, F. E. Rougieux, and D. Macdonald, Appl. Phys. Lett. 103, 092105 (2013). 